\title{
LA EVOLUCIÓN DEL CONCEPTO DE ACTOR EN LA TEORIA DE LAS RELACIONES INTERNACIONALES ${ }^{1}$
}

\author{
Caterina García Segura \\ Profesora de Relaciones Internacionales \\ Facultat de Ciencies Politiques i de Sociologia \\ Universitat Autònoma de Barcelona
}

Resumen

Este articulo pretende trazar un breve recorrido a través de las principales modificaciones y ampliaciones teóricas del concepto de actor internacional, es decir, reflejar las diferentes respuestas que se han ido ofreciendo a la pregunta de ¿QUIEN consigue ejercer su influencia en la esfera internacional? o, dicho de otra manera, ¿CUALES son los ACTORES del sistema internacional?.

Tomando como punto de partida el concepto realista de actor internacional, se irán introduciendo las criticas que las aproximaciones posteriores al realismo le han ido dirigiendo.

En primer lugar se recogerán las aportaciones de las aproximaciones que a partir de los años setenta reflejaron el fenomeno transnacional. El estado, actor por excelencia, y las organizaciones internacionales gubernamentales dejaron de ser considerados los unicos actores internacionales y fueron incluidas bajo ese concepto entidades como las empresas multinacionales, las organizaciones no $\mathrm{gu}^{\mathrm{u}}$ bernamentales, la opinión pública, los grupos de presión o los individuos.

A continuación se analizarán las aproximaciones que desde los años ochenta se han hecho eco del fenomeno subestatal a nivel internacional. Es decir, aquéllas que ponen especial énfasis en la importancia de la actividad exterior de las entidades políticas subestatales, tales como los estados federados, las comunidades autónomas, Las regiones o los municipios.

Finalmente, a través de las criticas al concepto realista de actor internacionaly de sus sucesivas ampliaciones, se presentarán los criterios necesarios para formular una definición operativa y comprehensiva de actor internacional que permita interpretar la complejidad de la sociedad internacional contemporánea.

Abstract

The purpose of this article is to follow the main theoretical changes and enlargements of the concept of international actor. In other words, to reflect the

1. Este artículo está basado en parte del trabajo realizado por la autora en su resis doctoral, La presència de les entitats politiques subestatals a les Relacions Internacionals, dirigida por Esther Barbé y leída en la Universicat Aurònoma de Barcelona en 1992. 
«Papers»: Revista de Sociologia

different answers given to the question WHO manages to influence on the international scene? or WHICH are the ACTORS of the international system?

Taking the realist concept of internatioal actor as a starting point, we will present the main criticisms from the subsequent approximations of the international relations theory.

Firstly, we will analyze the contributions from the approximations that, starting in the seventies, reflected the transnational phenomenon. The state, the main actor, and the intemational organizations were no longer considered the only actors and this concept started to include other entities as multinational corporations, non governmental organizations, public opinion, lobbies or individuals.

Secondly, we will analyze the approximations, starting in the eighties, that have taken into account the substate phenomenon at the international level, namely those which stressed the attention on the external activity of the substate political entities like federated states, comunidades autónomas, regions or cities.

Finally, through the criticisms to the realist concept of international actor and its following enlargements, we will present the reguired criteria to formulate an operational and comprehensive definition of international actor that allows us to interpret the complexity of the contemporary international society.

EI intentar dar respuesta a la pregunta ¿QUIÉN consigue ejercer su influencia en la esfera internacional?, es decir, ¿CUÁLES son los ACTORES del sistema internacional? ha sido una constante en la evolución teórica de la disciplina de las relaciones internacionales. La cuestión ha sido y sigue siendo clave en el debate teórico, puesto que de las repuestas que se le dan derivan las diferentes aproximaciones al estudio de las relaciones internacionales.

La aproximación dominante durante más tiempo en las relaciones internacionales ha sido el realismo. Éste, en base a los actores, se define como estatocéntrico, siendo considerado el estado como EL actor por excelencia de las relaciones internacionales. A pesar de que en el momento actual se admite claramente el pluralismo teórico y metodológico a nivel de las relaciones internacionales, el realismo estatocéntrico ha seguido ejerciendo un peso determinante. En consecuencia, todas las ampliaciones del concepto de actor han tenido que enfrentarse a dicha visión estatocéntrica.

A nivel de la realidad internacional, desde el final de la Segunda Guerra Mundial la escena internacional ha asistido a la multiplicación, en núrnero y variedad, de los actores que intervienen en ella. Desde la multiplicación del número de estados como consecuencia del proceso descolonizador hasta la de las empresas multinacionales como resultado de la mundialización de la economía, pasando por la proliferación de las organizaciones internacionales, gubernamentales y no gubernamentales, todas las ampliaciones sucesivas del número de actores internacionales han obedecido a la evolución y a la dinámica del sistema internacional contemporáneo.

A partir de los años setenta esta diversificación, cuantitativa y cualitariva, 
adquirió nuevas dimensiones. Este hecho tuvo repercusiones evidentes en la Teoría: la aproximación realista de las relaciones internacionales, imperante desde los años cincuenta, vio su dominio amenazado. Nuevas aproximaciones, insatisfechas con el carácter estatocéntrico del realismo, intentaban ofrecer nuevas respuestas que, naturalmente, incluían la toma en consideración de nuevos actores internacionales.

Desde la perspectiva de los actores internacionales, dos aproximaciones teóricas han intentado suceder al realismo.

En primer lugar la que, en los años setenta, desafió al realismo político apostando por el predominio de los actores transnacionales y por la ampliación de la gama de interacciones que desarrollan en el sistema internacional: el transnacionalismo.

En segundo lugar, aquéllas que, en los años ochenta, han pretendido ampliar el concepto de actor, añadiendo una dimensión subestatal a las categorías apuntadas por los transnacionalistas.

De este modo, el concepto de actor ha ido evolucionando, con el retraso lógico de la teoría respecto a la práctica, a medida que lo hacía el sistema internacional.

Desde la perspectiva de este estudio, el punto de partida que nos interesa es la afirmación realista, según la cual las relaciones internacionales son relaciones exclusivamente interestatales. Para Morgenthau (1948), máximo representante del realismo norteamericano, el estado es el único actor digno de consideración en un medio, como el sistema internacional, de carácter político, es decir, basado en el poder, porque es la forma histórica de organización del ejercicio del poder en las relaciones internacionales.

A pesar de las importantes diferencias entre los estados, éstos son considerados legal e igualitariamente soberanos (art. 2, p.l de la Carta de las Naciones Unidas) y ésta es la característica (jurídica) que les concede el monopolio en el escenario internacional. La realidad ha cuestionado estas premisas, y el debate endógeno sobre la crisis del estado-nación en un mundo interdependiente ha tenido sus efectos evidentes sobre el proceso de revisión del concepto de actor. Si bien puede decirse que el estado ha salido vencedor de esta lucha consigo mismo, el debate sobre los actores internacionales prosigue. Ya no se trata de cuestionar el protagonismo del estado, sino de afirmar teóricamente la presencia de los llamados "nuevos actores".

A continuación procederemos a analizar los principales términos del debate sobre la ampliación del concepto de actor internacional, a través de aquellas aproximaciones tẹóricas más significativas desde el punto de vista de nuestro objetivo.

En primer lugar, revisaremos la concepción estatocéntrica de las relaciones internacionales a través de sus críticos. 
En segundo lugar, analizaremos las dos aproximaciones que proponen la modificación del concepto de actor internacional: la ampliación del concepto a nivel transnacional y a nivel subestatal.

En tercer lugar, y a partir de los elementos de cambio apuntados, intentaremos formular los criterios necesarios para flegar a una definición operativa del concepto de actor dentro del actual contexto internacional.

\section{LAS CRITTCAS A LA APROXIMACION REALISTA DE LAS RELACIONES INTERNACIONALES}

La concepción realista de las relaciones internacionales se adecuaba perfectamente a las características del contexto en que se gestó: los Estados Unidos de la post-Segunda Guerra Mundial. Su pesimismo latente y el pragmatismo que propugnaban lo convirtieron en la aproximación más idónea para interpretar las relaciones internacionales propias de los momentos duros de la guerra fría.

Pero en los años setenta, las circunstancias internacionales habían cambiado, la distensión había sucedido a la guerra fría y los temas de seguridad y defensa comenzaban a ocupar un segundo plano en una agenda internacional, cada vez más cargada de temas de carácter económico-social. A nivel de actores, los estados se habían multiplicado y diversificado, a la vez que otras entidades intervenían activamente en la escena internacional.

Sin embargo, las interpretaciones realistas no habian evolucionado en parafelo a la sociedad internacional que pretendian interpretar. De ahí la crítica general que en los años setenta se dirige al carácter estatocéntrico del realismo: su incapacidad operacional para analizar e interpretar la realidad internacional. En realidad se puede hablar de una crítica en tres dimensiones diferentes.

1) El primer gran grupo de juicios respecto a la falta de pertinencia del reatismo se producen en torno a los atributos de universalidad y de permanencia, que se asignan al estado-nación desde las formulaciones teóricas realistas.

Desde la perspectiva realista, la definición de actor, a partir de la universalidad de la defunición de estado, agrupa bajo el mismo concepto a una serie de entidades muy distintas entre sí. El realismo ignora la diversidad de realidades que se acogen bajo el concepto de estado-nación. Aunque se establezcan jerarquías, entidades que representan realidades completamente diferentes, en base a su definición juridica, siguen formando parte de la misma categoría y, por tanto, son consideradas "actores internacionales".

Situar a priori bajo la misma consideración a estados que han ido desarroIlando sus estructuras político-administrativas y económico-sociales a través de largos procesos de formación y consolidación nacional y a otros que han sur- 
gido como resultado de procesos de independencia más o menos urgentes y más o menos dirigidos, no deja de ser un proceso, en cierta medida, deformador de la realidad; considerar actor a un estado por el simple hecho de poseer el reconocimiento juridico internacional y no hacerlo, o situar en una pseudocategoría subordinada, a una multinacional que realiza operaciones de un extremo a otro del planeta, de la que depende el empleo de miles de trabajadores y de cuyas decisiones puede depender en parte el nivel de vida que un estado pueda ofrecer a sus ciudadanos (y por tanto influir en el grado de cohesión social, de conflictividad laboral, de apoyo u hostilidad hacia el gobierno, etc.), tampoco parece conformarse a la dinámica del sistema internacional.

Por otra parte, el realismo, salvo contadas excepciones, presenta al estado como una realidad eterna o, simplemente, no se plantea la posibilidad de un cambio. El estado es una forma de organización territorial del poder político que, de la misma manera que surgió en un momento determinado, puede desaparecer o transformarse cuando deje de ser funcional. Tal como expresa Merle (1991), el estado no ha existido siempre. No es más que una forma de gestión del poder entre muchas otras. Taylor (1984), que sitúa al estado en el primer lugar de la jerarquía de actores internacionales, alega que no existe ninguna base histórica a partir de la cual asumir que el estado se mantendrá eternamente en el lugar que ahora ocupa.

2) El segundo grupo de críticas hace referencia al hecho de que el realismo define el concepto de actor en base a atributos de tipo jurídico que no reflejan la dinámica de las relaciones internacionales.

Refiriéndose a los estados como actores internacionales, Merle (1991) afirma que son las únicas entidades beneficiarias de pleno derecho de la plenitud de competencias concedidas a los sujetos del derecho internacional (derecho de legación, derecho de concertar tratados, derecho de guerra, derecho de impartir justicia).

Autores críticos con el realismo (Barber y Smith, 1984) afirman que la dimensión jurídica (soberanía e independencia), implícita en la definición de actor internacional, supone que sólo los estados pueden poseer dichos atributos. Dichos autores se muestran en desacuerdo con tal interpretación, en base a que la complejidad del sistema internacional de los años setenta desafía la arbitraria definición que identifica exclusivamente a los actores internacionales con los estados. Señalan la existencia en la escena internacional de entidades que actúan independientemente, con impacto internacional, y que, al no poseer el atributo de soberanía, no se incluyen en la definición clásica de actor internacional.

Los autores que consideran insuficiente el análisis realista proponen enfocar la cuestión de los actores internacionales desde la perspectiva del proceso 
"Papersn: Revista de Sociologia

de las interacciones que se dan en el espacio internacional, en lugar de hacerlo desde la perspectiva jurídico-formal. Así, lo que determinará la calificación de actor internacional será la dinámica del proceso por el que una entidad es capaz de movilizar una serie de recursos para alcanzar unos objetivos concretos y ejercer una influencia sobre otros actores del sistema.

En consecuencia, la autonomía sucederá a la soberanía como categoría de análisis. Es decir, se valorará la habilidad de una entidad para provocar consecuencias en la política internacional, sin que su comportamiento sea predecible a partir de la referencia a otros actores. Evidentemente se parte de la constatación que, en el sistema internacional contemporáneo, ningún actor es completamente autónomo.

Keohane y Nye (1973) también insisten en la importancia de la capacidad o de la habilidad versus la definición o calificación de entidad soberana: cuando entidades no estatales son capaces de afectar el curso de los acontecimientos internacionales se convierten en actores $y$, por tanto, entran en competencia con el estado-nación.

También la definición de actor internacional de Mansbach, Ferguson y Lampert (1976) deja al margen los atributos de soberanía y de independencia, centrándose en la capacidad de actuación. Para ellos, el principal problema del modelo estatocentrista es la creencia de que los estados soberanos agotan el universo de los posibles actores relevantes en la política global. Consideran que un actor no debe ser definido ni en base a la calidad adscriptiva de soberanía ní en base a la calidad descriptiva de territorialidad. Contrariamente, debe definirse a partir del atributo comportamental de autonomía.

3) Un último aspecto centra la crítica al estatocentrismo realista: la repercusión de la nueva agenda internacional en la formulación del concepto de actor.

Desde la perspectiva realista, la consideración de la agenda internacional se mantendría inalterable a pesar de los cambios experimentados por el sistema internacional de postguerra y, por tanto, no habría necesidad de introducir nuevas entidades en la categoría de actores. Ahora bien, si consideramos una gama más amplia de interacciones, nos veremos abocados a la ampliación de las entidades consideradas. Es lo que demuestra, justamente, el trabajo empírico de análisis detallado de la actividad internacional que llevan a cabo diversos autores (Rosenau, 1980; Mansbach et al.1976) ${ }^{2}$. Tras su investigación, los

2. Rosenau (1980:58ss) utiliza las noricias recogidas en la prensa de Los Angeles como evidencia del cambio experimentado tanto a nivel de agenda como a nivel de acrores. Mansbach ct al. (1976) realizaron un estudio empírico consistente en la recogida y análisis de datos extraidos del WEIS (World Event/Interaction Survey), del CREON (Comparative Research on the Events of Nations) y del New York Times durante un periodo de 25 años. Para una explicación 
trabajos mencionados aplican la categoría de actor a aquellas entidades que llevan a cabo tareas globales en una o más áreas temáticas (protección física, desarrollo y regulación económica, actividades públicas o que contribuyan a la cohesión social, entre otras) (Mansbach y Vasquez, 1981:7).

\section{LA FORMULACION TRANSNACIONALISTA DE LAS RELACIONES INTERNACIONALES}

Como el realismo político, la aproximación transnacionalista también es fruto de su época. Los cambios en el sistema internacional, la paz y la expansión económica impulsaron la articulación de nuevas relaciones $y$, a partir de los años setenta, quedó manifiesta la realidad de un mundo cerrado e interdependiente.

Los "padres del transnacionalismo" - Robert O. Keohane y Joseph S. Nye - empezaron a trabajar sobre el fenómeno transnacional a raíz de la constatación de los intereses coincidentes de los académicos de las diferentes disciplinas en el estudio de los actores no estatales. En su aproximación al tema, se planteaban todos los problemas a los que se enfrentaba el investigador en relaciones internacionales cuando intentaba explicar la realidad de los años setenta con los útiles realistas.

Keohane y Nye (1977) decidieron ir más allá del planteamiento de la problemática y de la revisión del estado de la investigación en relaciones internacionales, y se propusieron formular una teoría que superara las limitaciones del realismo. Sus motivaciones eran tanto de orden teórico como práctico: creían que muchas de las limitaciones de la política exterior estadounidense tenían su origen en las limitaciones de las premisas realistas en que se inspiraba. A pesar de ello sus objetivos eran puramente teóricos: pretendían llegar a la comprensión de la política internacional y, para ello, consideraban que en primer lugar debían ser capaces de aprehender la naturaleza cambiante del sistema internacional.

La primera transformación teórica que desde la perspectiva de este estudio nos interesa resaltar, es la del concepto de politica mundial (world politics) que utilizan los autores transnacionalistas. Ésta comporta el abandono de la idea de política internacional como lucha por el poder en términos político-militares. Se propone una concepción de la política mundial en la que el fenómeno central es la negociación entre una variedad de actores autónomos y semiautónomos (Keohane y Nye, 1973:380). Si el objetivo central deja de ser la maximización del poder en términos político-militares, otras entidades distintas de los estados pasarán a ocupar un lugar central en las relaciones internacionales.

metodológica profundizada de su NOSTAC (Nonstate Project), vid. el Apéndice de su obra (pp.301-317). 
"Papers": Revista de Sociologia

Su concepción teórica introduce tres cambios conceptuales que tendrán una repercusión directa en el desarrollo del concepto de actor.

1) La sustitución del concepto de lucha por el de negociación. En su opinión, en un universo nuclear la negociación se convierte en el proceso primordial de la política mundial. El objetivo no es el único ítem en base al que ha de calcularse y organizarse la estrategia. Los costes pasan a ser considerados muy relevantes. La interdependencia compcle a la negociación. El hecho de que las decisiones no se tomen en el vaćlo, que puedan tener consecuencias no controlables y que alteren cotalmente su eficacia, aconsejan el compromiso.

2) La ampliación de los escenarios en los que se desarrolla la política mundial y las interacciones juzgadas relevantes. Desde la formulación transnacional, el escenario político-militar pierde la exclusividad. En los setenta resultaba imposible no rcconocer la magnitud y la influencia política de materias originarias de otros campos y que se lievan a término sin el control gubernamental.

Los autores señalan cuatro tipos de interacciones globales que pueden tener significación en la política mundial: (1) las relacionadas con las comunicaciones: intercambio y movimiento de información, transmisión de ideas, creencias, etc.; (2) las relacionadas con los transportes y los movimientos físicos de objetos y bienes (personales y comerciales); (3) las interacciones en el ámbito financiero: movimientos de dinero y de créditos; (4) los viajes (movimientos de personas). A partir de esta consideración, la gama de interacciones de significación política es mucho más dilatada que en concepciones anteriores. Algunas de estas interacciones son iniciadas bajo dirección gubernamental, otras sólo lo son parcialmente y otras escapan completamente a su control.

La política mundial será concebida como el conjunto en el que se integran las relaciones interestatales, las relaciones intergubernamentales, y las relaciones transnacionales. las primeras coinciden con la actividad diplomática convencional y sus unidades son los estados y sus subunidades. Las segundas son las que se dan entre les organizaciones internacionales gubernamentales o sus subunidades ${ }^{3}$. Las últimas son las relaciones que tienen lugar entre las organizaciones transnacionales, sus subunidades y ciertos individuos ${ }^{4}$.

3. Keohane \& Nye (1973:xix y 381), en general, al hablar de la actuación internacional de "subunidades" del estado se refieren a ciertas "disidencias" por parte de algún ministerio (utilizan el ejemplo del "club" de ministros de Agricultura comunitarios y de sus bacallas contrá los respectivos miristros de Finanzas, o las presiones de los departamentos de metereología nortcamericanos y canadiense sobre sus tespectivos departamentos de estado para conseguir $\mathrm{l}_{\mathbf{a}}$ ampliación de los presupuestos de investigación en esta materia).

4. Keohane y Nyc (1973:xi) las definen como aquellos contactos, coaliciones c inceracciones a través de las fronteras que no están controladas por los órganos centrales de la política cxterior de los gobiernos centrales. 
La importancia de la consideración de tales relaciones va ligada al hecho de que el estado, actor internacional por excelencia, puede quedar excituido de determinadas relaciones relevantes a nivel internacional.

Los autores afrrman que las relaciones transnacionales no son nuevas. La novedad consiste en su consideración teórica. Su inclusión en el marco de reflexión de la política mundial, permitirá comprender y explicar el sistema internacional y las interacciones que se dan en él, más allá de lo permitido por el esquema realista.

3) La ampliación de los objetivos en política exterior y su carácter circunstancial permite que determinadas entidades, que no intrevendrían si los objctivos internacionales se limitasen a objetivos de poder político-militar, se conviertan circunstancialmente en actores al sentirse motivados por otro tipo de objetivos.

Todo ello lleva a la consideración del incremento del número y el tipo de actores internacionales. En efecto, los cambios introducidos hasta el momento nos conducen a la admisión de un mayor número de actores internacionales y de una mayor heterogeneidad de categorías. Se implicarán en asuntos internacionales "nuevos" actores (organismos y conferencias internacionales interactuarán directamente con los ministerios internos en materias especializadas) y también actores internos se convertirán cn "nuevos" actores internacionales (en base a la creciente experiencia exterior de algunos departamentos internos y de su mayor capacidad paza entender y coordinar determinados tipos de actividades al margen del ministerio de Exteriores).

Las pautas en base a las que una entidad del sistema puede considerarse actor serán diferentes a las que regían en la aproximación realista. Los requisitos serán de tipo funcional (en lugar de jurídicos). Funcionalidad, provisionalidad, capacidad de movilizar recursos y habilidad para crear solidaridades, lealtades $\mathrm{c}$ identificaciones serán algunas de las claves que flevarán al desplazamiento ocasional del estado de su posición central del escenario internacional.

El transnacionalismo permite incluir toda una amplia gama de entidades bajo el concepto de actor internacional, son todas aquellas que a lo largo de los años setenta se manifestaron activas sobre la escena internacional: empresas multinacionales, grupos de presión, organizaciones no gubernamentales, ministerios o agencias gubernamentales, etc.

A partir de los años ochenta y con un enfoque diferente del transnacionalismo, aparecen una serie de trabajos teóricos sobre actores internacionales que han centrado su atención en un fenómeno que se ha extendido notoriamente en la última década: el fenónemo subestatal. 
"Papers": Revista de Sociologia

\section{EL PARADIGMA DEL SISTEMA GLOBAL}

El paradigma del sistema global, también llamado Issue Paradigm, parte de las mismas críticas al realismo que las de los transnacionalistas. Su particularidad estriba en ir más lejos que las anteriores en la consideración de las categorías de actores, en precisarlas más.

Otro aspecto que to distingue es el enfoque que permite considerar interacciones apenas contempladas por los transnacionalistas, más preocupados por temas más en boga en el momento en que escribian (por ejemplo, la acción de las multinacionales). Mansbach y sus colaboradores no se centran en los actores internacionales de tipo económico, sino en los actores políticos subestatales. Las multinacionales habían adquirido protagonismo a causa de la expansión económica en unos años en que el crecimiento parecía ilimitado. Las entidades políticas subestatales se convertirán en actores importantes con la certeza de los límites del crecimiento y ante las dificultades de los estados para responder a los retos de la interdependencia y a las demandas económicosociales cada vez más apremiantes de los ciudadanos.

Los estudios de Mansbach y sus colaboradores tenían como objetivo detectar a los actores presentes en la escena internacional. Para ello iniciaron sus investigaciones a partir de la pregunta: "¿quién hace qué a quien en política global?m. Para poder responder organizaron el proyecto de investigación denominado Nonstate Actor Project (NOSTAC)

De las observaciones de sus estudios derivaron una serie de conclusiones:

1) A partir de la constatación de que en un $63,8 \%$ de todos los acontecimientos recensados intervenían actores no estatales, el reconocimiento

5. Metodológicamente, se proponían una sistemarización del comportamiento en base a los acontecimientos observados en la escena internacional. Los acontecimientos fueron utilizados como las unidades comparables necesarias para la construcción y comprobación de las reorias empíricas.

Para su estudio detcrminaron que serian consideradas acontecimientos las acciones que sólo tuvicran un actor $y$, como mínimo, un objetivo. Su comportamicnto debía ser observable y haber sido recogido por las fuentes utilizadas. Si un actor tenia más de un objetivo se lo clasificaba varias veces formando pareja con cada uno de ellos. Acotaron la investigación temporalmente 1948-1972. Clasificaron los dacos geográficamente según la región desde la que eran organizadas las accioncs aunque tuvieran lugar en otra parte. Clasificaron les noticias recogidas según categorias de comportamiento: actos cooperativos, conflictivos o participativos e intenciones cooperativas a conflictivas.

Una vez recogidos los datos fueron confrontados y establecieron una reclasificación inductiva de los actores. Con el cruce de los datos obtenidos y su análisis, intentaron dar respuesta a una serie de preguntas: ¿cuáles fueron las usuper-cuestiones" que dominaton la política de cada región en el período estudiado?, ¿cuál fue el ranking de importancia de los "super-temas" para cada fracción de tiempo determinada?, ¿cuál era la acritud de los accores (cooperación/conflicto, iniciativa/respuesta)? (Mansbach et al, 1976:68-69, 300 et ss.). 
de la importancia que éstos adquieren bajo circunstancias determinadas.

2) Ligada a la primera, la constatación de importantes diferencias regionales en cuanto a la cantidad y a la calidad de la actividad de los actores. El dinamismo de los actores no estatales varía mucho más que el de los estados según las regiones. Concluyen que hay un componente regional importante (tradición política, desarrollo económico) en la participación de un mayor o menor número de actores, en su grado de implicación y en su diversidad: las regiones menos desarrolladas (política y económicamente) se caracterizan por un mayor predominio de actuaciones estatales.

3) De la observación del comportamiento de los actores, concluyeron que el proceso político es único (rechazo de la división externo/interno) y que en él se dan dos tipos de interacciones: las interacciones de actores "dentro" de entidades más vastas o las interacciones de estas entidades "entre" sí.

4) Del análisis de todos los acontecimientos censados concluyeron que la agenda internacional es de carácter cambiante. Pueden existir muchos objetivos y valores muy diferentes en su base. Lo que es importante es poder identificar cuándo y cómo los objetivos se convierten en políticos ${ }^{6}$ y cuando se les concede un reconocimiento internacional. Los autores afirman que los individuos y los grupos empiezan a vincularse en cuanto descubren que comparten intereses (condición de similitud) y objetivos (condición de interdependencia) que transcienden las fronteras existentes.

La reflexión teórica que realizan a partir de sus estudios empíricos les conduce a la formulación de una nueva propuesta paradigmática, el Yssue Paradigm, que dé cabida y situé en el lugar apropiado a unos actores hasta entonces depreciados o infravalorados.

La primera consecuencia que comporta la adopción de su visión del mundo y de las relaciones internacionales es, desde la perspectiva que nos interesa, la ampliación del concepto de actor internacional. Mansbach y Vasquez afirman que los actores no están determinados por su adscripción jurídica a dicha categoría (posesión del atributo de soberanía), sino que lo serán sobre una base empírica. Los actores deben poseer una cierta medida de autonomía, unidad, capacidad y objetivos comunes. Desde el momento en que los recursos necesarios para ser influyentes varían de un tema a otro, la calidad de actor no es

6. Lo que distingue un actor políico de orros grupos es, según los autores cirados, la búsqueda de la satisfacción de las necesidades de sus miembros a través la utilización del sistema politico (Mansbach $y$ Vasquez, 1981:144-145). 
inherente a un determinado grupo de colectivos o de individuos. El atributo de actor está únicamente ligado a los objetivos y a las capacidades para intentar conquistarlos que tengan los miembros específicos de cada categoría general. En definitiva, la funcionalidad y la operatividad son la clave.

Por otra parte, las realidades de la interdependencia, de la penetración y la perforación de frọteras demuestran que un actor internacional no tiene nada que ver con un agregado de personas que vive dentro de unos límites geográficos o habla una misma lengua (aunque ello pueda facilitar una acción conjunta o un acuerdo en la determinación de valores y objetivos). El atributo de territorialidad deja de ser relevante y exclusivo al mismo tiempo que el de soberanía. Un actor, en cambio, ha de gozar del acceso centralizado a los recursos de los miembros, ha de ser capaz de ejercer un control sobre ellos y ha de ser receptor de sus lealtades.

El paradigma del sistema global propone una tipologia de actores que permite integrar a todos los considerados hasta el momento. Su propuesta incluye seis categorías:

(1) Actores gubernamentales interestatales (OIGs), es decir, las organizaciones conocidas como internacionales o supranacionales. Las OIGs son entidades que se caracterizan por: 1) derivar de un acto jurídico creador - tratado, carta, estatutos, etc.- que determina su organización y finalidad; 2) estar compuestas por sujetos de derecho internacional; 3) estar dotadas de órganos permanentes que son distintos e independientes de los miembros de la organización; 4) formar una voluntad colectiva a través de los órganos referidos, que es jurídicamente distinta a la de los miembros; 5) tener un carácter estable y permanente (Díez de Velasco, 1984:35).

(2) Actores no gubernamentales interestatales, también llamados fuerzas transnacionales. Esta categoría se refiere a los grupos e individuos que no representan a los estados en que se ubican. Las fuerzas transnacionales pueden definirse como los movimientos y las corrientes de solidaridad de origen privado que tratan de establecerse a través de las fronteras y que tienden a hacer valer o a imponer su punto de vista en el sistema internacional, por ejemplo, las organizaciones no gubernamentales (ONGs) o las empresas multinacionales (Merle, 1991:411).

(3) Estados-nación. Incluye la actuación de parcelas del gobierno, es decir, del personal de ministerios y agencias. Los autores insisten en resaltar el "particularismo" o "personalismo" de las decisiones "estatales" para contrastarlo con la idea del interés nacional incuestionable para los realistas.

(4) Actores gubernamentales no centrales. Aquí se sitúa el personal de los gobiernos locales (regionales, municipales) de un estado o los funciona- 
rios coloniales que representan a la metrópolis. Señalan que, habitualmente, estas burocracias locales están sólo periféricamente afectadas por la política mundial, pero que ocasionalmente pueden tener un impacto directo, ya sea cuando sirven de vehículo a aspiraciones secesionistas, ya sea cuando establecen contactos con otros actores.

(5) Actores intraestatales no gubernamentales. Son los individuos o grupos no gubernamentales que, ubicados en un estado, mantienen relaciones directas con otros actores autónomos distintos a su gobierno (organizaciones filantrópicas, partidos políticos, sindicatos, corporaciones industriales, etc.) y situados en el exterior de su estado.

(6) Individuos. Cuando, a nivel privado, son capaces de actuar autónomamente en la arena global.

Elaboran una matriz con todas las interacciones posibles entre estos seis tipos de actores, formando un sistema conglomerado complejo (complex conglomerate system). Este sistema constituye una propuesta explicativa de las relaciones internacionales, alternativa de las interpretaciones bipolares o multipolares, ya que, según estos autores, las explicaciones a partir de la estructura bipolar o multipolar de la distribución del poder en el sistema internacional sólo son válidas para los estados.

El término "conglomerado" hace referencia a la mezcla de materiales y de elementos diversos que se agrupan sin asimilarse. La idiosincrasia, el rasgo primario de este sistema, es la existencia de diversos tipos de actores diferentes que se agrupan según las situaciones específicas y forman distintos alineamientos difusos y flexibles en base a los objetivos que se pretenden alcanzar y a los medios que deben movilizarse para ello.

\section{LA APROXIMACION LOCAL-DEMOCRATIZADORA DE ALGER}

Otra aportación teórica que nos interesa destacar es la de Chadwick Alger. Alger (1977/1980/1990) también forma parte del grupo de autores que intentan romper con el modelo estatocéntrico. Parte para ello del esquema de Mansbach y lo amplía, incluyendo los niveles regionales y locales, tanto para las instancias gubernamentales como para las no gubernamentales.

La prerension de Alger era superar aquello que, desde su punto de vista, no consiguieron Keohane y Nye - abandonar la lógica estatocéntrica como principio articulador del sistema internacional-e ir todavía más lejos que Mansbach y sus colaboradores.

Alger reflexiona movido por la convicción política de la posibilidad de cambio del sistema. Postula la necesidad de ser conscientes de la resistencia al 
cambio que se opone desde el esquema de análisis del sistema internacional. Según Alger, si los periódicos no conceden mayor importancia a los actores subestatales - aludiendo a las investigaciones sobre actores internacionales realizadas a partir de las noticias que aparecen en ellos - no es porque no actúen significativamente en el sistema, sino, precisamente, por la pauta que domina la consideración de lo que debe o no ser estimado susceprible de ser noticia.

La postura de Alger es, en este aspecto, más militante que teórica a favor del cambio del sistema internacional a partir de valores diferentes a los tradicionalmente asignados al estado o a las multinacionales. Sin negar el peso de la economía global en la estructuración de las nuevas interacciones transnacionales, apunta hacia una categoría de motivaciones diferentes.

Orienta sus investigaciones desde el nivel local. Estudia las ciudades como unidades de análisis de la política mundial. Justifica el impacto de las ciudades en la política global con los siguientes argumentos: 1) son núcleos creadores de una nueva tecnología y una cultura que después se extenderá más allá de ellos; 2) son puntos de intersección (nodes) de los sistemas internacionales que brindan facilidades para su conexión; 3) son los cuarteles generales desde donde se controlan tanto el sistema internacional gubernamental, como el no gubernamental; 4) la gente se identifica con ellas y se dirige a ellas en búsqueda de protección y apoyo.

Cuando el autor analiza las relaciones internacionales de las ciudades incluye dos grupos de razones que explican su actividad internacional: el impacto de los procesos económicos y sociales de alcance mundial (paro, vivienda, contaminación, etc.) y la respuesta por parte de ciudadanos a nivel local a temas tradicionales de la política exterior estatal (movimientos ciudadanos contra el apartheid, ciudades desnuclearizadas, movimientos en favor de los derechos humanos, etc.).

Desde nuestro punto de vista, este último bloque de objetivos de carácter humanitario, aun siendo importante, no constituye todavía un impulso significativo para la acción internacional de las ciudades o de otros actores gubernamentales no centrales. Nuestra afirmación se basa en la consideración del carácter aún minoritario e ideológico de este tipo de temáticas, mienrras que los impacros de la globalización de la economía son motivos más universales para la movilización de los actores gubernamentales no centrales.

En segundo lugar, las motivaciones aducidas por Alger suponen una capacidad de respuesta y/o de enfrentamiento directo al sistema, una voluntad explícita de cambio, en definitiva, una clara iniciativa por parte de las ciudades que no se da en la realidad. Actualmente, las actuaciones gubernamentales no centrales a nivel internacional todavía son mayoritariamente respuestas a los retos lanzados desde el sistema. Más que intentos de cambio son intentos de adecuarse a él lo más satisfactoriamente posible, de sacar el mayor número de ventajas. 
Por otra parte, son las unidades políticas subestatales de los países más industrializados las que tienen una mayor capacidad de respuesta o mayores posibilidades de planificar su actuación (control de medios, disponibilidad de información, etc.). Sin embargo, su actuación internacional no está motivada por una discrepancia ideológica con la política del gobierno central. Más bien. lo que intentan es actuar directamente a nivel internacional, a fin de evitar la desviación o demora de sus objetivos en el proceso de canalización a través de las instancias del gobierno central.

\section{LA REFLEXION TEORICA SOBRE LAS «SOBERANIAS PERFORADAS»}

Una tercera aportación teórica dirigida a ampliar, directa o indirectamente, el concepto de actor internacional y a reformular los criterios de su conceptualización es la formulada por Duchacek, Latouche y Stevenson a finales de los ochenta. Estos autores elaboran el concepto de usoberanias perforadas para explicar el fenómeno de la penetración de las fronteras nacionales, presentándolas asi como tamices (sieves) frente a la imagen realista de las fronteras como caparazones (hard shells) impenetrables. Su orientación se fundamenta en el intento de denunciar el contenido falaz del término "soberanía". La interdependencia acaba con los compartimentos estancos y los comportamientos de los actores empiezan a ser jaspeados, marmoleados, (marbled), en el sentido de que se ven interferidos, penetrados, fragmentados o coordinados por o con los de orros actores.

Las soberanías perforadas son el rasgo característico de las fronteras actuales. Este es uno de los cambios mayores del sistema y en su base está la necesidad de adaptación y no nuevos valores como sugería Alger.

Esta necesidad tiene una doble vertiente: la económica (mencionada ya tantas veces) y la étnica (referida al despertar de los grupos étnicos dispersos en varios estados soberanos o concentrados en estados, provincias, comunidades autónomas o regiones, dentro de un estado soberano).

A partir de aquí, sus estudios se centran en un tipo de "perforadores de las soberanías", los "perforadores subnacionales". Justifican su elección con un dato cuantitativo: el $40 \%$ de la población mundial vive bajo sistemas federales, y una tercera parte de la población mundial lo hace bajo sistemas que aplican principios federales y tienen gobiernos que utilizan formas de organización similares a las federales (citan los ejemplos de Bélgica y España). El hecho numérico no hace sino reforzar la explicación del protagonismo actual de este tipo de relaciones y de la participación más activa de los actores gubernamentales no centrales.

Distinguen cuatro categorías de "perforadores subnacionales": 
1) La oposición (sea democrática o revolucionaria). Los gobiernos extranjeros se ven compelidos a considerar su actuación en base a la posibilidad de toda oposición de converrirse en gobierno.

2) Los grupos de intereses privados (las empresas que llegan a desarrollar una actividad transnacional, los sindicatos, los media, etc.).

3) Los inmigrantes (en su calidad de fuerza laboral).

4) Los gobiernos locales, municipales o regionales, especialmente las comunidades territoriales o etno-territoriales.

Estos perforadores adoptan dos formas de participación internacional. El primer tipo de actuación va dirigido a influir en el proceso de decisión del gobiemo central (lobbies que dirigen sus esfuerzos hacia las decisiones del legislativo o del gobierno central). El segundo tipo de actuación se concentra en evitar los mecanismos del gobierno central mediante el mantenimiento de contactos directos transgubernamentales, formales o informales, con actores próximos geográfica o funcionalmente.

\section{CRITERIOS PARA UNA DEFINICION OPERATIVA DEL CONCEPTO DE ACTOR INTERNACIONAL}

El conjunto de aproximaciones teóricas examinadas hasta aquí nos permite llegar a la formulación de nuevos criterios para definir un actor internacional. La definición que podrá derivarse de estos criterios deberá ser una definición que permita analizar e interpretar la realidad internacional contemporánea. Esto implica que ha de ser capaz de permitir la consideración de todos los grupos, entidades, colectivos e individuos que participan activamente en el sistema internacional, así como de todas las interacciones que tienen lugar en él.

Estos criterios aparecen, explícitamente, a lo largo de las críticas dirigidas a la aproximación estatocentrica de las relaciones internacionales o se desprenden, implícitamente, de las mismas. Si bien algunos autores prefieren definiciones más restrictivas y limitan, conscientes de excluir algunas categorías, los criterios de su definición ${ }^{7}$, o establecen sus criterios en base al estado ${ }^{8}$, aquí se apunta hacia criterios amplios.

7. Por ejemplo, Taylor (1984:20-22) reconoce que su defrnición de actores internacionales no estatales es restrictiva, pero la adopta porque afirma que, dada la diversidad de estos actores, hoy en día él prefiere concentrarse en aquellos que son relarivamente más amplios y más permanentes.

8. Russer y Searr (1989:50) señalan como criterios para la consideración de una entidad como accot internacional: 1) que realice funciones significativas y continuadas en el sistema in- 
El primer criterio a considerar sería el de utilizar un enfoque funcional opuesto al jurídico imperante. No importa el reconocimiento jurídico internacional de una entidad, sino su habilidad para movilizar determinados recursos para la consecución de determinados objetivos y su capacidad para ejercer influencia sobre el comportamiento de otros actores del sistema internacional.

Una orientación basada en la actitudy en el comportamiento de los actores debería substituir a la orientación legalista basada en los atributos jurídicos establecidos a priori. La calidad de actor debería establecerse en base a variables de comportamiento observables y no en base a aptitudes establecidas por definición.

El segundo criterio que debería ser introducido es el de la relatividad y la temporalidad de la condición de actor. La consideración de una entidad, grupo o individuo como actor internacional puede cambiar según las circunstancias. Ningún actor, ni ningún tipo de actor es eterno.

Ligado a los anteriores y complementario, el tercer criterio: la diversidad. Con el relevo de la soberanía por la autonomía conquistaremos la posibilidad de considerar como actores internacionales a categorías muy diversas $y$, lo más innovador, podremos admitir como tales a entidades, grupos o individuos detentores de diferentes grados de autonomía.

Cuando hablábamos de soberanía estábamos ante actores iguales. Podía haber jerarqufas de poder entre los actores, pero, en esencia, se trataba de actores reconocidos como idénticos por el derecho internacional. Estábamos ante sujetos con "personalidad internacional", es decir, sujetos con derechos y deberes ante el derecho internacional. Los acontecimientos de los últimos cuarenta años nos aconsejan la admisión como actores internacionales de toda una serie de entidades, colectivos y/o individuos que poseen, o pueden poseer, diferentes capacidades para actuar autónomamente. Probablemente, la capacidad para actuar autónomamente de la que gozará una gran multinacional norteamericana, europea o japonesa no tendrá ni punto de comparación con la que se le pueda presumir a un estado soberano en vías de desarrollo.

Lo mismo es aplicable a las entidades políticas subestales. Por ejemplo, California, estado federado de los Estados Unidos, no es una entidad soberana y su actividad exterior se enfrenta a constricciones que no afectan a la de los Estados Unidos. Pero si consideramos su economía en términos absolutos, California, con 29 millones de habitantes, se sitúa en el sexto lugar en el ranking mundial de PNBs globales (cogidos los de los estados miembros de los

temacional, 2) que su actividad sea relevante para los decisores de la política exterior del estado $y$, por tanto, influyente en su formación y 3) que haya atgún grado de auconomia y libertad en su toma de decisiones. Puntualizan que éstos y otros criterios definen a los actores en términos del actor dominante, el estado. 
Estados Unidos separadamente del PNB del conjunto de la federación). Esto la sitúa por encima de países como Italia, el Reino Unido o Canadá9.

Entre los veinticinco primeros puestos de dicho ranking hallariamos diex estados federados. Entre los cincuenta PNBs globales más aitos, hay treinta y tres correspondientes a estados federados. Entre los setenta y cinco primeros, cincuenta pertenecen a entidades políticas subestatales.

Según cual sea la esfera de actuación considerada, la cuestión o el tipo de problemática, la actividad exterior de California como actor económico internacional, puede ser mucho más relevante que la de un estado miembro de $\mathrm{Na-}$ ciones Unidas. Esta situación, en un sistema de interdependencia compleja en el que se da una distribución sectorial del poder, es cada vez más real.

El mismo esquema puede aplicarse a otro tipo de actores gubernamentales no centrales: las ciudades, por ejemplo. Así, Los Angeles ocuparía el decimotercer lugar en el citado ranking de PNBs por delante de países como Australia, Suiza o Austria.

Con la misma lógica, podemos afirmar que ha sido significativo para Sudáfrica el comportamiento de diversas ciudades norteamericanas que han prohibido a las empresas que tienen en ellas sus sedes mantener relaciones comerciales y/o industriales con dicho país. Así, Sudáfrica se ha visto más afectada por la actitud negativa hacia su régimen de un determinado gobierno local, que por la actitud positiva de un estado-nación con el que no tenía ningún interés económico compartido.

En definitiva, todos los criterios a considerar para llegar a formular una definición operativa de actor internacional conducen a la afirmación de la necesidad de centrar el análisis en el proceso, en la dinámica de las interacciones que tienen lugar en el escenario internacional y no en una concepción estática del sistema internacional.

La teoría debe estar abierta a los sucesivos cambios del sistema, reflejar las tendencias que se van imponiendo y no estar al servicio de la perpetuación de una determinada visión del mundo.

En el marco de los noventa, con la experiencia de las últimas décadas y los nuevos daros aporrados por el resurgir de los nacionalismos europeos a partir de la caída del muro de Berlín y de la desintegración de la URSS, creemos que los criterios enunciados permiten cubrir la gama de actores que intervienen en la arena internacional contemporánea. No afirmamos que todos estén presentes en la misma medida ni de la misma manera, pero sí que todos ellos -desde los más consolidados teóricamente hasta los introducidos más recientemente- pueden desempeñar actuaciones de relevancia internacional.

9. Estos datos, así como los citados en los ejemplos siguientes, han sido extraidos de la comunicación de Earl H. Fry (1990:4). 


\section{BIBLIOGRAFIA CITADA.}

Alger, Ch. (1977), "The Impact of Cities on International System", Eukistics, vol. 44, núm. 264, pp. 243-253.

Alget, Ch. (1988), "Los nexos locales-mundiales: su percepción, análisis y enfoque", Revista Internacional de Ciencias Sociales, núm. 117, pp. 339-360.

Alger, Ch. (1990), "The World Relations of Cities: Closing the Gap Between Social Science Paradigms and Everyday Experiencen, Intemational Studies Quarterly, vol. 34, núm. 3, pp. 493-518.

Barber, J. \& Smith, M. (1984), The nature of foreign policy. A reader, Edimburg, Holmes McDougall, Open University Press.

Diez de Velasco, M. (1984), Instituciones de Derecho Internacional Priblico, tomo II, Organizaciones Internacionales, Madrid, Tecnos.

Duchacek, I., Latouche, D. \& Stevenson, G. (1988), Perforated Sovereignities and International relations. Trans-sovereign Contacts of Subnational Governments, Westport, Greenwood Press.

Fry, E.H. (1990), The New International Cities Era: Case Studies from the United States, comunicación presentada en la Universidad Menéndez Pelayo, Valencia, 25-29 de junio de 1990.

Keohane, R.O. \& Nye, J.S. (1973), Transnational Relations and World Politics, Cambridge, Harvard University Press, (1 a edición, 1971).

Keohane, R.O. \& Nye, J.S. (1977), Power and Interdependence, Boston, Little, Browun \& Company.

Mansbach, R.W., Ferguson, Y.H. \& Lampert, D.E. (1976), The Web of World Politics: non-State Actors in the Global System, Englewood Cliffs, Prentice-Hall.

Mansbach, R.W. \& Vasquez, J.A. (1981), In Search of Theory. A New Paradigm for Global Politics, New York, Columbia University Press.

Merle, M. (1991), Sociologia de las relaciones internacionales, Madrid, Alianza Editorial.

Rosenau, J. (1980), "Muddling, Meddling and Modeling: Alternative Approaches to the Study of World Politics in an Era of Reapid Change", en Misra K.P. \& Smith Beal, R. (eds.), International Relations Theory, Western and non-Western Perspectives, Nueva Delhi, Vikas Publisihong House, pp. 57-79.

Russet, B. \& Starr, H. (1989), World Politics. The Menu for Choice, San Francisco, W.H. Freeman and Company, (1 ${ }^{2}$ edición, 1984).

Taylor, P. (1984), Nonstate Actors in International Politics: From Transregional to Substate Organizations, Boulder and London, Westiew Press. 